\title{
3 Research Square

\section{Evaluation of mandibular inferior cortical bone thickness using panoramic radiographs in edentulous and dentate cases}

\section{Reza Derafshi}

Shiraz University of Medical Sciences

Janan Ghapanchi

Shiraz University of Medical Sciences

Mitra Farzin

Shiraz Unversity of Medical Scineces

Abdolaziz Haghnegahdar

Shiraz University of Medical Sciences

Maryam Zahed ( $\nabla$ maryamzhd@yahoo.com )

Shiraz University of Medical Sciences Faculty of Dentistry https://orcid.org/0000-0001-5047-4018

Maedeh Tavakoli

Shiraz University of Medical Sciences

Research article

Keywords: Dentition, Edentulous Jaw, Osteoporosis, Panoramic Radiography

Posted Date: July 19th, 2019

DOI: https://doi.org/10.21203/rs.2.11693/v1

License: (c) (i) This work is licensed under a Creative Commons Attribution 4.0 International License.

Read Full License 


\section{Abstract}

Background: Osteoporosis is a disease of the bone structure which has many outcomes for the patient. Tooth loss and failure of implant placement can be related to this disorder in the jaw bones which is shown by the reduction of mandibular inferior cortical thickness. The aim of this study is to find a relationship between mandibular inferior cortical thickness using panoramic radiographs and tooth existence. Methods: A total of 57 panoramic views of complete edentulous patients and 164 partial edentulous cases were evaluated and compared to 117 dentate age and sex matched subjects. Mandibular cortical thickness was measured on 3 reference points (below the mental foramen (S1), estimated position of the first molar (S2) estimated position of the third molar (S3)) on both left and right sides using marking gauge in Agfa program. Results: Data revealed that cortical bone thickness was significantly reduced in older patients $(P=0.031)$. The final value of the three measurements of the mandibular border thickness was $2.3128 \mathrm{~mm} \pm 0.74840$ in the right side (RS) and $2.407 \pm 0.802 \mathrm{~mm}$ in the left side (LS) for the complete edentulous group, $2.9026 \pm 0.7513 \mathrm{~mm}$ in RS and $2.9976 \pm 1.06769$ in LS for partial edentulous group and 2.8709 \pm 0.6263 in RS and $2.9812 \pm 0.83081$ in LS for the dentate cases. Complete edentulous cases and partial edentulous cases that had lost posterior teeth had no significant difference in cortical thickness, but both groups had a significant thinner cortical width compared to dentate subjects $(P=0.001$ and $P=0.002)$. But lack of anterior teeth did not have the same effect compared to dentate individuals $(P=0.929)$. Conclusions: The present study demonstrates a significant relationship between tooth loss in the posterior region and reduction of inferior mandibular border which is a value of osteoporosis. Anterior tooth loss however does not have the same effect. Dentist and physicians should be in close contact in such patients to avoid further tooth loss and also diagnose this chronic disease in earlier stages.

\section{Background}

Osteoporosis happens when bone mineral density is reduced, resulting in the increase likelihood of fractures. Due to the aging of worldwide populations there is an increase in the incidence of this asymptomatic bone disease. Currently this disorder is one of the major public health concerns for the elderly populations. (1)

One of the main consequences of this bone disease is bone fracture which unfortunately occurs before identifying the individuals who have osteoporosis. $(1,2)$ Thus, detection of osteoporosis, assessment of bone mass, and identification of fracture risk are essential when evaluating the older population especially postmenopausal women.(2) Osteoporosis is usually defined by bone mineral density (BMD) measurements, known as a T-score. Osteoporosis is diagnosed in women by the World Health Organization according to the age and sex-matched average as a bone mineral density of 2.5 standard deviations below peak bone mass.(3) Literature reveals that two of five women have risk of osteoporosis. (3) 
Diagnosis of osteoporosis is important due to fracture risks, especially in high risk groups such as postmenopausal women.(4) However, BMD tests are very expensive, especially in developing countries; therefore they are not commonly used for the early detection of fracture risks. (5)

Several investigators suggest that panoramic radiograph or orthopanthomographs (OPG) of the head and neck region could be a useful identification tool in individuals with low BMD scores.(6-8) By careful and professional examination of panoramic radiographs, the change in quality and quantity of bone can be determined.(7) Previous studies have stated that osteoporosis results in altered structure of the inferior border of the mandible.(7-9)

Panoramic radiography is routinely obtained because of its capability to demonstrate a comprehensive view of the maxillofacial structure and dentition. Although dentists mainly use this radiography to focus on teeth and jaw anomalies, it can help medical practitioners to evaluate the overall systemic health of the patient. (10)

A number of mandibular cortical indices, including the mandibular cortical index (MCI), mandibular cortical width (MCW) and panoramic mandibular index (PMI), have been developed to assess osteoporosis and osteopenia by evaluating the quality of mandibular bone mass in panoramic radiographs. $(6,11)$ The best introduced measurement is the mental index (MI), which is the mean width of the mandibular inferior border cortex below the mental foramen.(11) Osteopenia in the jaw bones is defined as the thinning of the cortex at the lower border of the mandible. Also a reduction in bone mineral density is related to thin mandibular cortical width. $(12,13)$ In a recent study, the correlation between MI, age and BMD at the lumbar spine, femoral neck and total hip were evaluated. The results showed that thickness and morphological changes of mandibular inferior cortical bone are associated with BMD at the lumbar spine, femoral neck and total hip.(8) The indices in another study indicated that there is an area in the mandibular cortex, located between the mental foramen and the antegonial region, which is suitable for identifying females at high risk for osteoporosis. (14)

Moreover, several studies have stated that decreased skeletal BMD is also associated with premature tooth loss. $(15,16)$ Such patterns of tooth loss are consistent with periodontal bone loss and osteoporotic status. (15-17) Nevertheless, as indicated in literature there is no definite evidence of association between tooth loss and osteoporosis.(17)

Therefore, in this study we aimed to assess the cortical bone thickness of the mandibular inferior border in dentate, partial edentulous and complete edentulous patients and to compare these indices in age and sex matched individuals to better understand the relationship of teeth existence and osteoporosis. To the best of our knowledge this comparison has not been previously investigated. 


\section{Methods}

\section{Study group}

This analytic cross-sectional study was conducted on 221 panoramic views (114 female, 107 male, and aged 33-83) of patients who used either a partial denture (partial edentulous) or a full mouth denture (complete edentulous). All cases had been referred to Shiraz Medical University dental clinic for routine oral examination. A control group of 117 dentate healthy subjects (50male ,67 female ,aged 38-80 years old, who visited the same clinic for routine dental care), between February 2017 to August 2018 , were retrieved to determine the thickness of inferior mandibular cortex .Control group was matched with the cases regarding sex and age. Radiographs of acceptable quality entered the study. A written-informed consent from was obtained from all participants. Individuals with the history of radiotherapy, any metabolic bone disease (hyperparathyroidism, hypoparathyroidism, Paget's disease, osteomalacia, renal osteodystrophy, or osteogenesis imperfecta), cancers with bone metastasis, significant renal impairment, anemia, corticosteroid and anti-osteoporosis medication or any medication that affects bone metabolism, such as estrogen, and a history of smoking were excluded from the study. Demographic data including name, gender, age and duration of denture use was registered for all patients in a prearranged form. The study was approved by the ethical committee of Shiraz University of Medical Sciences.

\section{Methods}

Panoramic views were prepared by a planmeca XC prolin panoramic machine (Helsinky, Finland). Exposure factors were adjusted according to the size and age of the patients (57785 kvp,10 mA), using an Agfa PSP receptor (Germany).

All radiographs were evaluated by an expert in oral and maxillofacial radiology and a last year student using magnification device and proper viewer.

By drawing a tangent line with the inferior border of mandible, cortical thickness was measured on 3 reference points (below the mental foramen (S1), estimated position of the second molar (S2) estimated position of the third molar (S3)) on both left and right sides using marking gauge in Agfa program (Figure1 and 2). In dentate subjects the apex of the second premolar (S1), apex of the second molar (S2) and middle of the condyle and coronoid process (S3) was used as reference points. The mean value of the measured three points was mentioned as the final value. We have used the same method in our previous study to evaluate osteoporosis in certain dentate individuals with systemic disease.(12) Also this method has been used in other studies to assess osteoporosis of the jaws. $(18,19)$

Figure1: The measurements of the inferior mandibular cortex in a partial edentulous subject in three points, below the mental foramen, estimated position of the first molar, estimated position of the third molar. 
Figure 2: The measurements of the inferior mandibular cortex in a complete edentulous subject in three points, below the mental foramen, estimated position of the first molar, estimated position of the third molar.

\section{Statistical analyses:}

All data were collected and analyzed using SPSS software (version 18; SPSS Inc, Chicago, IL, USA). To compare the 3 index points, repeated measure ANOVA test was employed. Student's t test was performed to show the relation between age groups and mandibular cortical thickness. The mean mandibular cortical thickness in both groups was evaluated by One-way ANOVA and Tukey test. An error probability ( $p$ value) of $<0.05$ was accepted as significant.

\section{Results}

A total of 57 panoramic views of complete edentulous patients (mean age 62.80 \pm 9.86), 164 partial edentulous (mean age 58.53 \pm 11.506 ) and 117 dentate subjects (mean age 56.83 \pm 6.841 ) were reviewed in the oral and maxillofacial radiology clinic of the University of Shiraz, Iran. The partial edentulous group consisted of 94 cases who had lost posterior teeth of both sides (57\%), 25 cases had posterior teeth and lacked anterior teeth (15.2\%) and 46 cases had lost posterior teeth of either left or right side (27.8\%). Demographic data of the cases are listed in table 1.

Data revealed that cortical bone thickness was significantly reduced in accordance with aging regardless of teeth existence. Older individuals demonstrated lower cortical bone thickness $(\mathrm{p}=0.031)$. But sex had no significant relationship with cortical thickness. $(\mathrm{P}=$ 0.671)

The mean three values (S1, S2, S3) registered for each group is mentioned in table 1. In complete edentulous group the final value of the three measurements of the mandibular border thickness was $2.3128 \mathrm{~mm} \pm 0.74840$ in the right side (RS) and $2.407 \pm 0.802 \mathrm{~mm}$ in the left side (LS), for all partial edentulous patients regardless of the area of tooth loss the mean amount was $2.9026 \pm 0.7513 \mathrm{~mm}$ in RS and $2.9976 \pm 1.06769$ for LS. For dentate control group this amount was $2.8709 \pm 0.6263$ in RS and $2.9812 \pm 0.83081$ for LS. (Table 2 and 3)

The mean cortical thickness was significantly lower in complete edentulous cases than the dentate groups $(\mathrm{P}=0.001)$. Moreover, partially edentulous cases who lacked posterior teeth on both sides showed a significantly thinner cortical bone than dentate age and sex matched individuals $(\mathrm{p}=0.002)$. In patients who had lost anterior teeth there was no significant difference between mandibular inferior cortex thicknesses in comparison to dentate cases. $(\mathrm{P}=0.929)$ 
When comparing complete edentulous cases and partial edentulous cases (missing posterior teeth on both sides) there was no significant difference between cortical bone thickness $(\mathrm{P}=0.086)$. But the mandibular inferior cortical bone measurements were significantly higher in the anterior teeth edentulous subjects in comparison to posterior edentulous and complete edentulous patients $(p=0.001)$ Furthermore, in the partial edentulous group, a significant correlation was not seen between the cortical thickness and missing teeth on one side or the anterior region $(p=0.347)$.

Table 1: Demographic information of dentate and edentulous subjects

\begin{tabular}{|c|c|c|c|c|c|c|c|}
\hline & \multicolumn{2}{|c|}{ Dentate } & \multicolumn{2}{|c|}{ Partial edentulous } & \multicolumn{2}{|c|}{ Complete edentulous } \\
\hline & & Number & Percentage & Number & Percentage & Number & Percentage \\
\hline \multicolumn{2}{|c|}{ Total } & 117 & $35 \%$ & 164 & $48.2 \%$ & 57 & $16.8 \%$ \\
\hline \multicolumn{2}{|c|}{ Age } & \multicolumn{2}{|c|}{$38-80$} & \multicolumn{2}{|c|}{$33-93$} & \multicolumn{2}{|c|}{$40-83$} \\
\hline \multirow[t]{2}{*}{ Gender } & Female & \multicolumn{2}{|r|}{67} & \multicolumn{2}{|r|}{81} & \multicolumn{2}{|r|}{31} \\
\hline & Male & \multicolumn{2}{|r|}{50} & \multicolumn{2}{|r|}{83} & \multicolumn{2}{|r|}{26} \\
\hline
\end{tabular}

Table 2: The mean measurements (S1, S2, S3) in millimeter of the inferior cortical mandibular thickness in cases and controls on the right side of the jaw

\begin{tabular}{|c|c|c|c|}
\hline \multirow{2}{*}{ Groups } & \multicolumn{3}{|c|}{ Mean thickness \pm SD } \\
\cline { 2 - 4 } & S1 & S2 & S3 \\
\hline Dentate & $3.74 \pm 1.08$ & $3.45 \pm 0.76$ & $1.36 \pm 0.60$ \\
\hline Partial edentulous & $3.84 \pm 1.27$ & $3.44 \pm 0.96$ & $1.31 \pm 0.53$ \\
\hline Complete edentulous & $2.88 \pm 1.19$ & $2.78 \pm 0.96$ & $1.13 \pm 0.30$ \\
\hline
\end{tabular}

Table 3: The mean measurements (S1, S2, S3) in millimeter of the inferior cortical mandibular thickness in cases and controls on the left side of the jaw

\begin{tabular}{|c|c|c|c|}
\hline \multirow{2}{*}{ Groups } & \multicolumn{3}{|c|}{ Mean thickness \pm SD } \\
\cline { 2 - 4 } & S1 & S2 & S3 \\
\hline Dentate & $3.80 \pm 0.95$ & $3.29 \pm 0.97$ & $1.46 \pm 0.78$ \\
\hline Partial edentulous & $4.04 \pm 1.20$ & $3.56 \pm 1.12$ & $1.24 \pm 0.49$ \\
\hline Complete edentulous & $3.26 \pm 1.16$ & $2.80 \pm 1.07$ & $1.17 \pm 0.64$ \\
\hline
\end{tabular}

\section{Discussion}


The main finding of this study was that edentulous subjects were suffering from thinner mandibular inferior cortex in comparison to dentate cases. Even though, older patients showed thinner mandibular cortex regardless of teeth existence, nevertheless cortical bone thickness was related to posterior teeth existence in this study. And what is more interesting is that the location of tooth loss (anterior vs. posterior) is highly related to low mandibular cortical indices. Lack of molar teeth results in lower measurements than anterior teeth. These findings have not been investigated formerly in literature as far as we have learnt.

In a finite element model it is exhibited that with a decrease in bone level in periodontal patients stress is increased on teeth especially on the apical region. And hyperocclusal loads are responsible for diseased periodontium.(20) Further studies have demonstrated this correlation in resistance to mechanical loading and bone mineral density. $(20,21)$ Thus, reduced bone mass and greater occlusal forces associated with posterior teeth has been suggested as a possible explanation for increased molar loss.(21) Our study also demonstrates that patients with missing teeth fall in the thinner mandibular bone cortex group. That is thin mandibular cortex can be an indicator of future tooth loss, although further studies are warranted to support this issue.

It is previously demonstrated that mandibular cortical bone is subjected to resorptive activity in osteoporotic individuals, resulting in decreased thickness and increased porous inferior border. $(22,23)$ Accordingly, the usefulness of cortical width and shape of the mandible in panoramic radiographs, for identifying osteoporosis in susceptible individuals, especially postmenopausal women is shown in studies. (22) A systematic review discovered that panoramic measurements demonstrates moderate diagnostic accuracy of hip fracture in osteoporotic patients compared to central dual energy X-ray absorptiometry (DXA) of the hip (24). Interestingly, studies reveal that cortical porosity is a less reliable predictor of osteoporosis than mandibular cortical width and in the evaluation of panoramic radiographs, only those patients with thin mandibular cortices should be referred for further osteoporosis investigation. (25) Various studies have evaluated mandibular indices to confirm osteoporotic changes of the jaw in susceptible patients throughout the years.(8, $9,11,12,14)$ In 2007 researchers suggested that measurement of mandibular cortical width is an alternative method of osteoporosis risk assessment, used for patients who have undergone radiography for usual dental reasons.(9) Following these findings, in 2010 seven panoramic values were evaluated for accuracy in osteoporosis diagnosis in comparison to bone mineral density in osteoporotic postmenopausal women. In this investigation Leite et al. showed that the most accurate indices were the mental index, mandibular cortical index, and visual estimation of cortical width. Antegonial indices and gonial angle are not good predictors in this matter.(11) Same results are seen in the study of Kim et al. who recently found that regardless of age, height and weight of the patient, thickness and morphological changes of mandibular inferior cortical bone are associated with BMD. (8). We also used 
the same methodology to detect osteoporosis in our previous research in 2017 on candidates of liver and kidney transplant. We found that patients with such systemic disease tend to have a thinner cortical width and they are more susceptible to osteoporotic changes. (12) Therefore, we also support the fact that bone resorption may easily be detected in this radiography, and panoramic indices such as thickness of the mandibular inferior cortex can be useful means to detect osteoporosis in older patients in comparison to expensive and invasive procedures. Moreover, posterior tooth loss can also be an early indicator of this disease according to our new findings in this study. Accordingly, patients who lose teeth or patients who are suffering from periodontal disease which results in tooth loss can be subjected to osteoporosis in the near future. Therefore, it is suggested that dentists should be aware of such patients and advise them for further evaluation of this chronic burden. Also patients with low mandibular inferior border thickness which is related to osteoporosis are subjected to loosing teeth. Therefore we propose that individuals that are suffering from this disease should be referred to dentists or dental hygienist as soon as possible to avoid further tooth loss.

Conversely, Gulsahi et al. found no correlation in bone mineral density (BMD) of mandible and maxilla compared to femoral BMD. They also debate the panoramic radiomorphometric indices and show no significant relationship with osteoporosis. (26) As they mention the sample size was small in that study and they had only evaluated edentulous subjects. This shows the importance of evaluating dentate patients next to edentulous subjects and comparing these individuals as we have done in the current study. This issue is supported in other studies who discuss the effect of teeth existence on bone density of the jaws.(15-17) Alveolar ridge dimensions in the buccolingual and also apicocoronal aspect are reduced as a consequence of a physiologic response after teeth extraction. (27) Moreover, there is evidence of low bone mineral density existence which results in tooth loss in patients.(15) In a study conducted by Darcey and colleagues the association of teeth number and osteoporosis was investigated. The researchers found that molar tooth loss has a significant relationship with the existence of osteoporosis. Therefore in conclusion they suggest that osteoporotic patients are at a greater risk of losing their teeth than healthy subjects and they are in need of more intensive preventive measures. (15) This is due to the resultant periodontal disease which is seen more prevalently in osteoporotic patients. (17) After comparing postmenopausal with premenopausal women with existing periodontitis, Svedha and colleagues found that periodontitis is a risk indicator for osteoporosis due to the significant increase in severe periodontal disease found in patients with existing osteoporotic conditions and vice versa.(16)

Evidently, dental implants are new modalities for replacing missing teeth. Regarding the quality of life in older osteoporotic patients a study revealed that in partially edentulous subjects implant placement augments life quality in comparison to fixed restoration, removable restoration and no restoration of missing teeth.(28) In spite of that, osteoporosis and low bone mineral density is a drawback in this treatment. There is still debate in the 
future of implant placement in osteoporotic patients. An increase in peri-implant bone loss was observed in this group. (29) Furthermore, it is observed that osteoporotic patients tend to have higher rates of implant loss compared to healthy individuals. Due to the matter that there are no randomized clinical trials to date to challenge this issue, still no final conclusion can be made. What is more, there are no evidence that osteoporosis might have effects on bone healing.(30) And yet there are no contraindications mentioned in literature for implant use in this group.(31) Therefore, according to the findings of our study which show thinner mandibular cortex in edentulous subjects, we support this issue that subjects who lose teeth, especially in the posterior region, should be placed on implant treatment as soon as possible to avoid further bone loss. This could augment the life quality of such patients.

Although cone beam computed tomography (CBCT) is a new modality for detecting precise textural changes of bone in the head and neck region, but in a recent research in 2018 panoramic and CBCT images exhibited good agreement in MI measurements and moderate to good agreement in PMI findings.(32) Hence, we also chose to evaluate panoramic radiography in our study in comparison to CBCT which is more expensive and not cost beneficial.

It is to note that, cross-sectional studies with this design only show exposure and prevalence of disease. As osteoporosis is a chronic disease it is likely that those with lower mandibular indices in this cross-sectional study were suffering from this condition for many years. Therefore, the effects can be somewhat variable in different individuals. So, it is difficult to assess the influence of disease throughout the years upon the outcome measures. More controlled prospective longitudinal studies would help in this assessment.

\section{Conclusion}

There is a significant correlation between tooth loss, aging and thinner mandibular cortex which is an indicator of osteoprosis, but the strength of this correlation needs further studies on larger groups. There is a statistically significant relationship between molar loss and mandibular inferior cortical bone thickness. And as it is shown anterior tooth loss does not have the same effect. It is more probable that local and other systemic factors exert great influence upon tooth loss which should be evaluated in further studies. Dentist should refer patients with periodontal disease and tooth loss for early osteoporosis detection. Moreover, physicians should be aware of tooth loss in such individuals. It is worthy to state that when planning a treatment like implant placement for a patient all disruptive measures like osteoporosis should be taken into account in advance to reduce the burden that is placed on the patient. 


\section{Declarations}

Ethics approval and consent to participate:

The study was approved by the ethical committee of Shiraz University of Medical Sciences. Consent for publication:

A signed consent form was taken from each patient in order to use their radiography in this paper.

Data Availability:

Other radiographies used to support the findings of this study are available upon request from the corresponding author.

Competing interests:

The authors declare that they have no competing interests.

Funding:

The authors thank the Vice-Chancellery of Shiraz University of Medical Sciences for supporting this research.

Authors' contributions:

$\mathrm{RD}, \mathrm{JG}, \mathrm{AH}, \mathrm{MZ}$ and MF have made contribution in the design of the work. MT, MF and JG were mainly involved with the patients and gathering data. AH and MT measured the radiographies. JG, $\mathrm{RD}$ and $\mathrm{MZ}$ interpreted and analyzed the data. MZ was mainly involved in drafting the manuscript. MZ and JG revised the manuscript and all authors have read and approved the final manuscript.

\section{Acknowledgements:}

This research is based on the thesis by Maedeh Tavakoli. We also thank Dr. Mehrdad Vosooghi of the Dental Research Development Center of the School of Dentistry for the statistical analysis.

\section{References}

1. Sözen T, Özışık L, Başaran N. An overview and management of osteoporosis. European journal of rheumatology. 2017;4(1):46-56.

2. Richards AM, Coleman NW, Knight TA, Belkoff SM, Mears SC. Bone density and cortical thickness in normal, osteopenic, and osteoporotic sacra. Journal of osteoporosis. 2010;2010.

3. Kanis JA. Diagnosis of osteoporosis and assessment of fracture risk. The Lancet. 2002;359(9321):1929-36. 
4. Kanis JA, Borgstrom F, De Laet C, Johansson H, Johnell O, Jonsson B, et al. Assessment of fracture risk. Osteoporosis international. 2005;16(6):581-9.

5. Silva BC, Broy SB, Boutroy S, Schousboe JT, Shepherd JA, Leslie WD. Fracture risk prediction by nonBMD DXA measures: the 2015 ISCD official positions part 2: trabecular bone score. Journal of Clinical Densitometry. 2015;18(3):309-30.

6. Kathirvelu D, Anburajan M. Prediction of low bone mass using a combinational approach of cortical and trabecular bone measures from dental panoramic radiographs. Proceedings of the Institution of Mechanical Engineers Part H, Journal of engineering in medicine. 2014;228(9):890-8.

7. Kavitha MS, An SY, An CH, Huh KH, Yi WJ, Heo MS, et al. Texture analysis of mandibular cortical bone on digital dental panoramic radiographs for the diagnosis of osteoporosis in Korean women. Oral surgery, oral medicine, oral pathology and oral radiology. 2015;119(3):346-56.

8. Kim OS, Shin MH, Song IH, Lim IG, Yoon SJ, Kim OJ, et al. Digital panoramic radiographs are useful for diagnosis of osteoporosis in Korean postmenopausal women. Gerodontology. 2016;33(2):185-92.

9. Karayianni K, Horner K, Mitsea A, Berkas L, Mastoris M, Jacobs R, et al. Accuracy in osteoporosis diagnosis of a combination of mandibular cortical width measurement on dental panoramic radiographs and a clinical risk index (OSIRIS): the OSTEODENT project. Bone. 2007;40(1):223-9.

10. Lim L, Padilla R, Reside G, Tyndall D. radiographic Features And Differential Diagnoses Of Pathology: Comparing Panoramic Radiographs And Cbct. Oral surgery, oral medicine, oral pathology and oral radiology. 2017;124(1):e29.

11. Leite AF, Figueiredo PT, Guia CM, Melo NS, de Paula AP. Correlations between seven panoramic radiomorphometric indices and bone mineral density in postmenopausal women. Oral surgery, oral medicine, oral pathology, oral radiology, and endodontics. 2010;109(3):449-56.

12. Ghapanchi J, Haghnegahdar AA, Faghih M, Rezazadeh F, Derafshi R, Farzin M, et al. Evaluation of mandibular inferior cortex changes in patients candidate for liver and kidney transplantation using panoramic view. Journal of Nephropathology. 2017;6(4).

13. Ghapanchi J, Zahed M, Haghnegahdar A, Niakan N, Sadeghzadeh A. Osteoporosis and Jaw Abnormalities in Panoramic Radiography of Chronic Liver Failure Patients. BioMed research international. 2018;2018.

14. Valerio CS, Trindade AM, Mazzieiro ET, Amaral TP, Manzi FR. Use of digital panoramic radiography as an auxiliary means of low bone mineral density detection in post-menopausal women. Dento maxillo facial radiology. 2013;42(10):20120059.

15. Darcey J, Horner K, Walsh T, Southern H, Marjanovic EJ, Devlin H. Tooth loss and osteoporosis: to assess the association between osteoporosis status and tooth number. British dental journal. 2013;214(4):E10.

16. Svedha PR, Mahendra J, Theayarajar R, Namachivayam A. Comparison of bone mineral density among pre- and post-menopausal women with and without chronic generalized periodontitis. Journal of Indian Society of Periodontology. 2017;21(2):119-24. 
17. Juluri R, Prashanth E, Gopalakrishnan D, Kathariya R, Devanoorkar A, Viswanathan V, et al. Association of Postmenopausal Osteoporosis and Periodontal Disease: A Double-Blind Case-Control Study. Journal of international oral health : JIOH. 2015;7(9):119-23.

18. Bollen A-M, Taguchi A, Hujoel PP, Hollender LG. Case-control study on self-reported osteoporotic fractures and mandibular cortical bone. Oral Surgery, Oral Medicine, Oral Pathology, Oral Radiology, and Endodontology. 2000;90(4):518-24.

19. Lee K, Taguchi A, Ishii K, Suei Y, Fujita M, Nakamoto T, et al. Visual assessment of the mandibular cortex on panoramic radiographs to identify postmenopausal women with low bone mineral densities. Oral Surgery, Oral Medicine, Oral Pathology, Oral Radiology, and Endodontology. 2005;100(2):226-31.

20. Reddy RT, Vandana KL. Effect of hyperfunctional occlusal loads on periodontium: A threedimensional finite element analysis. Journal of Indian Society of Periodontology. 2018;22(5):395400 .

21. Nakayama R, Nishiyama A, Shimada M. Bruxism-Related Signs and Periodontal Disease: A Preliminary Study. The open dentistry journal. 2018;12:400-5.

22. Gulsahi A. Osteoporosis and jawbones in women. Journal of International Society of Preventive \& Community Dentistry. 2015;5(4):263-7.

23. Yuzugullu B, Gulsahi A, Imirzalioglu P. Radiomorphometric indices and their relation to alveolar bone loss in completely edentulous Turkish patients: a retrospective study. The Journal of prosthetic dentistry. 2009;101(3):160-5.

24. Devlin H, Whelton C. Can mandibular bone resorption predict hip fracture in elderly women? A systematic review of diagnostic test accuracy. Gerodontology. 2015;32(3):163-8.

25. Devlin H, Karayianni K, Mitsea A, Jacobs R, Lindh C, van der Stelt P, et al. Diagnosing osteoporosis by using dental panoramic radiographs: the OSTEODENT project. Oral surgery, oral medicine, oral pathology, oral radiology, and endodontics. 2007;104(6):821-8.

26. Gulsahi A, Paksoy CS, Ozden S, Kucuk NO, Cebeci AR, Genc Y. Assessment of bone mineral density in the jaws and its relationship to radiomorphometric indices. Dento maxillo facial radiology. 2010;39(5):284-9.

27. Hansson S, Halldin A. Alveolar ridge resorption after tooth extraction: A consequence of a fundamental principle of bone physiology. Journal of dental biomechanics. 2012;3:1758736012456543.

28. DeBaz C, Hahn J, Lang L, Palomo L. Dental Implant Supported Restorations Improve Quality of Life in Osteoporotic Women. Int J Dent. 2015;2015:451923.

29. de Medeiros F, Kudo GAH, Leme BG, Saraiva PP, Verri FR, Honorio HM, et al. Dental implants in patients with osteoporosis: a systematic review with meta-analysis. International journal of oral and maxillofacial surgery. 2018;47(4):480-91.

30. Giro G, Chambrone L, Goldstein A, Rodrigues JA, Zenóbio E, Feres M, et al. Impact of osteoporosis in dental implants: A systematic review. World journal of orthopedics. 2015;6(2):311-5. 
31. Tsolaki IN, Madianos PN, Vrotsos JA. Outcomes of dental implants in osteoporotic patients. A literature review. Journal of prosthodontics : official journal of the American College of Prosthodontists. 2009;18(4):309-23.

32. Koseoglu Secgin C, Gulsahi A, Yavuz Y, Kamburoglu K. Comparison of mandibular index values determined from standard panoramic versus cone beam computed tomography reconstructed images. Oral surgery, oral medicine, oral pathology and oral radiology. 2018.

\section{Figures}

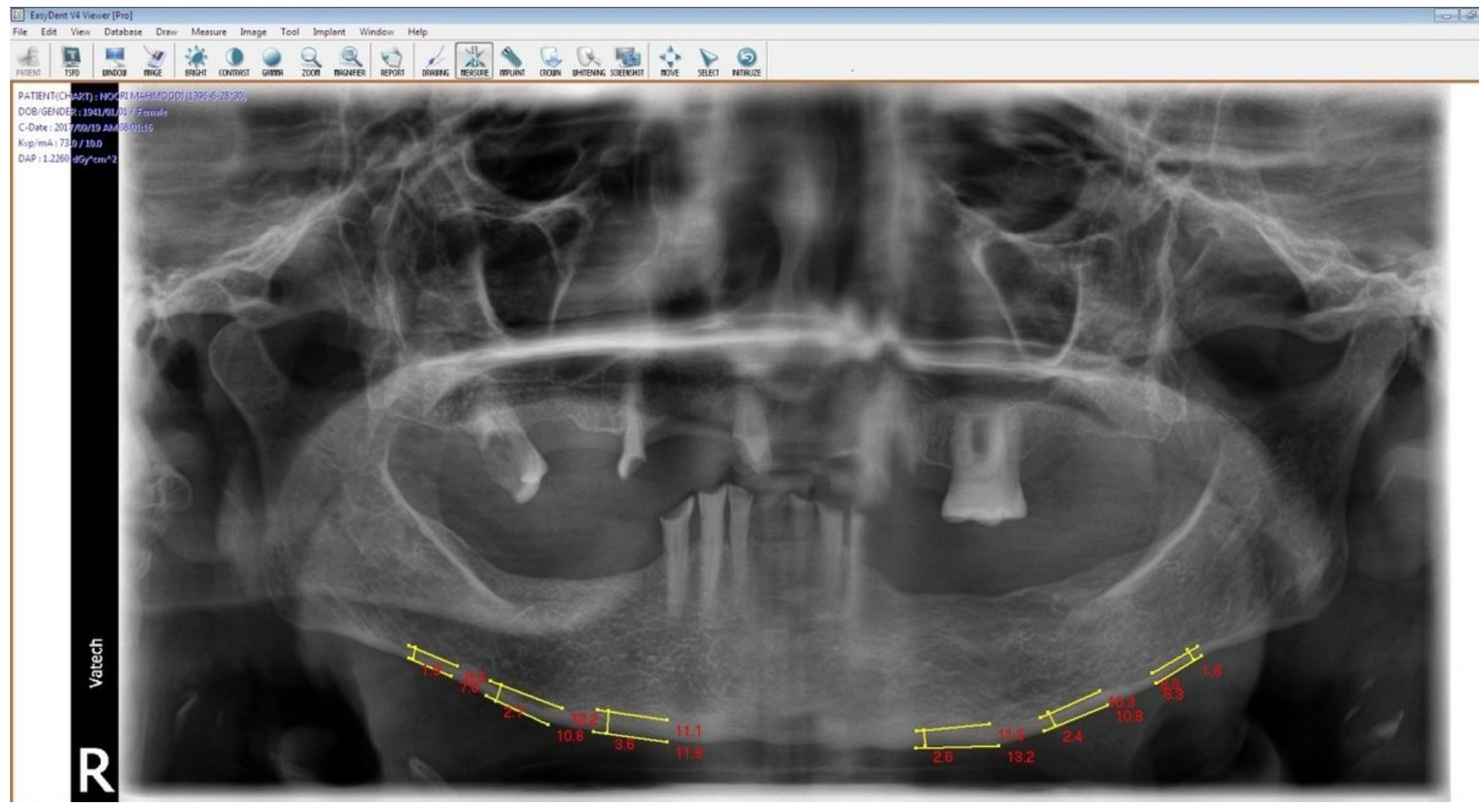

\section{Figure 1}

The measurements of the inferior mandibular cortex in a partial edentulous subject in three points, below the mental foramen, estimated position of the first molar, estimated position of the third molar. 


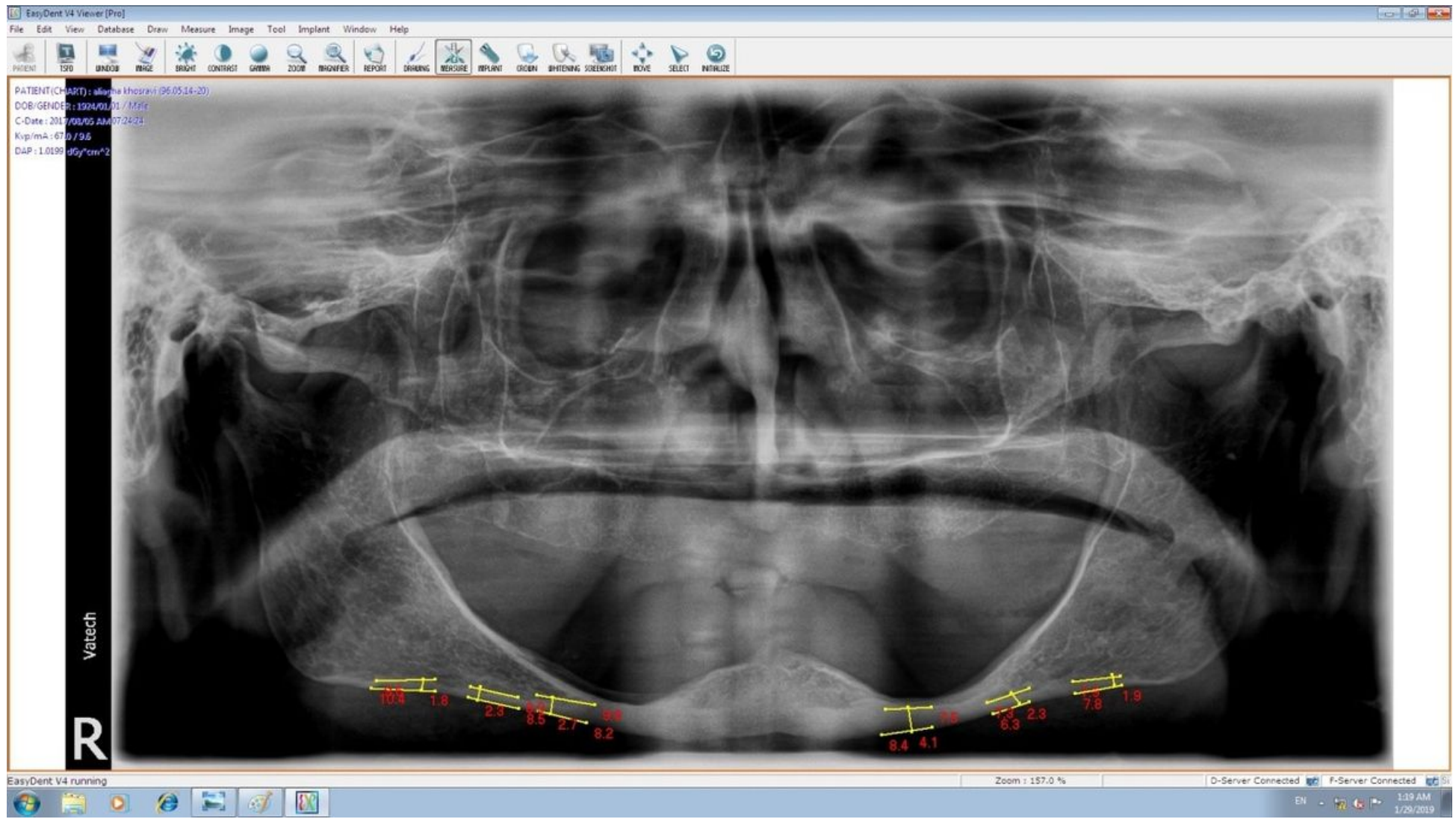

\section{Figure 2}

The measurements of the inferior mandibular cortex in a complete edentulous subject in three points, below the mental foramen, estimated position of the first molar, estimated position of the third molar. 